International Electronic Journal of Geometry

Volume 8 No. 2 PP. 57-69 (2015) (CIEJG

\title{
LIGHTLIKE HYPERSURFACES WITH PARALLEL SCREEN SHAPE OPERATOR
}

\author{
MAHAMANE MAHI HAROUNA, CYRIAQUE ATINDOGBE, JOËL TOSSA \\ (Communicated by Yusuf YAYLI)
}

\begin{abstract}
In this paper, we study lightlike hypersurfaces with parallel screen shape operator. The main result is a characterization theorem for lightlike with parallel screen shape of a Lorentzian space form.
\end{abstract}

\section{INTRODUCTION}

The theory of hypersurfaces, defined as submanifolds of codimension one, is one of the fundamental theories of submanifolds. As it is known, the main difference between the geometry of hypersurface in Riemannian manifold and in semiRiemannian manifold is that in the latter case the induced metric tensor field by the semi-Riemannian metric on the ambient space is not necessarily non-degenerate. If the induced metric tensor field is degenerate, the classical theory of Riemannian and semi-Riemannian hypersurfaces fails since the normal bundle and the tangent bundle of the hypersurface have a non zero intersection.

The main purpose of the present paper is to give a characterization of lightlike hypersurfaces with parallel screen shape of a Lorentzian space form. Section 2 covers useful preliminaries for study the geometry of lightlike hypersurfaces. In Section 3 , we prove that lightlike hypersurface $M$ with parallel screen shape operator is either totally geodesic or totally umbilic and if the screen is conformal, then $M$ is locally a lightlike triple product manifold (Theorem 3.1). At the end of section, we prove that results obtained in this paper are stable with any change of null section $\xi \in \operatorname{Rad}(T M)$.

\section{Preliminaries on Lightlike hypersurfaces}

Let $(\bar{M}, \bar{g})$ be a $(m+2)$-dimensional semi-Riemannian manifold of index $\nu,(0<$ $\nu<m+2)$. Consider a hypersurface $M$ of $\bar{M}$ and denote by $g$ the tensor field induced by $\bar{g}$ on $M$. We say that $M$ is a lightlike (degenerate, null) hypersurface if $\operatorname{rank}(g)=m$. Then the normal vector bundle $T M^{\perp}$ intersects the tangent bundle

Date: Received: July 14, 2014 and accepted: June 20, 2015.

2010 Mathematics Subject Classification. 53C20, 53C40, 53C50.

Key words and phrases. Lightlike hypersurface, screen conformal, screen shape operator.

The first author is supported by DAAD (Deutscher Akademischer Austausch Dienst). 
along a nonzero differentiable distribution called the radical distribution of $M$ and denoted by $\operatorname{Rad}(T M)$ :

$$
\operatorname{Rad}(T M): x \mapsto \operatorname{Rad}\left(T_{x} M\right)=T_{x} M \cap T_{x} M^{\perp} .
$$

A screen distribution $S(T M)$ on $M$ is a non-degenerate vector bundle complementary to $T M^{\perp}$. A lightlike hypersurface endowed with a specific screen distribution is denoted by the triple $(M, g, S(T M))$. As $T M^{\perp}$ lies in the tangent bundle, the following result has an important role in the study of the geometry of lightlike hypersurfaces.

Theorem 2.1. ([9]) Let $(M, g, S(T M))$ be a lightlike hypersurface of $(\bar{M}, \bar{g})$. Then there exists a unique vector bundle $\operatorname{tr}(T M)$ of rank 1 over $M$, such that for any non zero section $\xi$ of $T M^{\perp}$ on a coordinate neighborhood $\mathcal{U} \subset M$, there exists a unique section $N$ of $\operatorname{tr}(T M)$ on $\mathcal{U}$ satisfying

$$
\bar{g}(N, \xi)=1 \text { and } \bar{g}(N, N)=\bar{g}(N, W)=0,
$$

for all $W \in \Gamma\left(S(T M)_{\mid \mathcal{U}}\right)$.

With this theorem we may write the following decomposition

$$
T \bar{M}_{\mid M}=S(T M) \perp\left(T M^{\perp} \oplus \operatorname{tr}(T M)\right)=T M \oplus \operatorname{tr}(T M),
$$

where $\perp$ denotes an orthogonal direct sum and $\oplus$ a direct sum. Throughout the paper, we denoted by $\Gamma(E)$ the $C^{\infty}(M)$-module of smooth sections of a vector bundle $E$ over $\mathrm{M}$, while $C^{\infty}(M)$ represents the algebra of a smooth functions on $M$. Also, all manifolds are supposed to be smooth, paracompact and connected.

Let $(M, g, S(T M))$ be a lightlike hypersurface of a semi-Riemannian manifold $(\bar{M}, \bar{g}), \bar{\nabla}$ be the Levi-Civita connexion of $\bar{M}, \nabla$ the induced connection on $(M, g)$. Gauss and Weingarten formulas provide the following relations (see details in [9])

$$
\begin{aligned}
& \bar{\nabla}_{X} Y=\nabla_{X} Y+h(X, Y), \\
& \bar{\nabla}_{X} V=-A_{V} X+\nabla_{X}^{t} V,
\end{aligned}
$$

for all $X, Y \in \Gamma(T M)$ and $V \in \operatorname{tr}(T M)$, where $\nabla_{X} Y$ and $A_{V} X$ belong to $\Gamma(T M)$ while $h$ is a $\Gamma(\operatorname{tr}(T M))$-valued symmetric $C^{\infty}(M)$-bilinear form on $\Gamma(T M)$ and $\nabla^{t}$ is a linear connection on $\operatorname{tr}(T M)$. It is easy to see that $\nabla$ is a torsion-free connection. Define a symmetric $C^{\infty}(M)$-bilinear form $B$ and a 1-form $\tau$ on the coordinate neighborhood $\mathcal{U} \subset M$ by

$$
\begin{gathered}
B(X, Y)=\bar{g}(h(X, Y), \xi), \\
\tau(X)=\bar{g}\left(\nabla_{X}^{t} N, \xi\right)
\end{gathered}
$$

for all $X, Y \in \Gamma\left(T M_{j \mathcal{U}}\right)$. Then, on $\mathcal{U}$, equations (2.4) and (2.5) become,

$$
\begin{aligned}
& \bar{\nabla}_{X} Y=\nabla_{X} Y+B(X, Y) N, \\
& \bar{\nabla}_{X} N=-A_{N} X+\tau(X) N,
\end{aligned}
$$

respectively. It is important to stress the fact that the local second fundamental form $B$ in Eq.(2.8) does not depend on the choice of the screen distribution and satisfies,

$$
B(X, \xi)=0
$$


for all $X \in \Gamma\left(\left.T M\right|_{\mathcal{U}}\right)$. Let $P$ be the projection morphism of $T M$ to $S(T M)$ with respect to the decomposition (2.2). We obtain: for all $X, Y \in \Gamma(T M)$ and $U \in$ $\Gamma\left(T M^{\perp}\right)$,

$$
\begin{aligned}
\nabla_{X} P Y & =\stackrel{*}{\nabla}_{X} P Y+\stackrel{*}{h}(X, P Y), \\
\nabla_{X} U & =-\stackrel{*}{A} U_{U} X+\stackrel{*}{\nabla}^{t} U,
\end{aligned}
$$

where $\stackrel{*}{\nabla}_{X} P Y$ and $\stackrel{*}{A} U$ belong to $\Gamma(S(T M)), \stackrel{*}{\nabla}$ and $\stackrel{*}{\nabla}^{t}$ are linear connections on $\Gamma(S(T M))$ and $\Gamma\left(T M^{\perp}\right)$ respectively, $\stackrel{*}{h}$ is a $\Gamma\left(T M^{\perp}\right)$-valued $C^{\infty}(M)$-bilinear form on $\Gamma(T M) \times \Gamma(S(T M)),{ }^{*} A_{U}$ is a $\Gamma(S(T M))$-valued $C^{\infty}(M)$-linear operator on $\Gamma(S(T M)) . \stackrel{*}{h}$ and $\stackrel{*}{A}_{U}$ are the second fundamental form and the shape operator of the screen distribution $S(T M)$ respectively. Define on $\mathcal{U}$ the following relations

$$
\begin{aligned}
C(X, P Y) & =\bar{g}(\stackrel{*}{h}(X, P Y), N), \\
\epsilon(X) & =\bar{g}\left(\nabla^{t}{ }_{X} \xi, N\right) .
\end{aligned}
$$

One shows that $\epsilon(X)=-\tau(X)$. Thus, locally (2.11) and (2.12) become

$$
\begin{gathered}
\nabla_{X} P Y=\stackrel{*}{\nabla}_{X} P Y+C(X, P Y) \xi, \\
\nabla_{X} \xi=-\stackrel{*}{A}_{\xi} X-\tau(X) \xi,
\end{gathered}
$$

respectively. The linear connection $\stackrel{*}{\nabla}$ is a metric connection on $\Gamma(S(T M))$. But, in general, the induced connection $\nabla$ on $M$ is not compatible with the induced metric $g$. Indeed, we have:

$$
\left(\nabla_{X} g\right)(Y, Z)=B(X, Y) \eta(Z)+B(X, Z) \eta(Y),
$$

for all $X, Y \in \Gamma\left(\left.T M\right|_{\mathcal{U}}\right)$, where

$$
\eta(X)=\bar{g}(X, N),
$$

for all $Y \in \Gamma\left(\left.T M\right|_{\mathcal{U}}\right)$. Finally, it is straightforward to verify that

$$
\begin{aligned}
B(X, Y) & =g\left(\stackrel{*}{A}_{\xi} X, Y\right), \quad g\left(A_{N} Y, N\right)=0, \\
C(X, P Y) & =g\left(A_{N} X, Y\right), \quad \stackrel{*}{A_{\xi}} \xi=0,
\end{aligned}
$$

for $X, Y \in \Gamma\left(\left.T M\right|_{\mathcal{U}}\right)$.

We denote the curvature tensor associated with $\bar{\nabla}$ and $\nabla$ by $\bar{R}$ and $R$, respectively. Then we have ([9]): for all $X, Y \in \Gamma\left(\left.T M\right|_{\mathcal{U}}\right)$

$$
\begin{aligned}
& \bar{R}(X, Y) Z=R(X, Y) Z+A_{h(X, Z)} Y-A_{h(Y, Z)} X+\left(\nabla_{X} h\right)(Y, Z)-\left(\nabla_{Y} h\right)(X, Z), \\
& \begin{aligned}
g(R(X, Y) P Z, P W)= & g(\stackrel{*}{R}(X, Y) P Z, P W)+C(X, P Z) B(Y, P W) \\
& -C(Y, P Z) B(X, P W),
\end{aligned} \\
& \bar{g}(\bar{R}(X, Y) \xi, N)=C(Y, \stackrel{*}{A} \xi X)-C\left(X, \stackrel{*}{A}_{\xi} Y\right)-2 d \tau(X, Y) .
\end{aligned}
$$




\section{LIGHTLIKE HYPERSURFACES WITH PARALLEL SCREEN SHAPE OPERATOR}

In this section, we consider a lightlike hypersurface $M$ of a semi-Riemannian manifold $(\bar{M}(k), \bar{g})$ of constant curvature $k$. We need the following proposition.

Proposition 3.1. [2] Let $(\bar{M}(k), \bar{g})$ be a semi-Riemannian manifold of constant curvature $k$ and $M$ be a lightlike hypersurface of $\bar{M}(k)$. Let $R$ the curvature tensor of the induced connection $\nabla$ on $M$ by the Levi-civita connection $\bar{\nabla}$. For any $X, Y, Z \in \Gamma(T M)$, we have:
(a) $R(X, Y) Z=k\{g(Y, Z) X-g(X, Z) Y\}-B(X, Z) A_{N} Y+B(Y, Z) A_{N} X$;
(b) $\left(\nabla_{X} B\right)(Y, Z)-\left(\nabla_{Y} B\right)(X, Z)=B(X, Z) \tau(Y)-B(Y, Z) \tau(X)$;
(c) $B\left(A_{N} Y, X\right)-B\left(A_{N} X, Y\right)=2 d \tau(X, Y)$;
(d) $\left(\nabla_{Y} A_{N}\right)(X)-\left(\nabla_{X} A_{N}\right)(Y)+k\{\eta(X) Y-\eta(Y) X\}=\tau(Y) A_{N} X-\tau(X) A_{N} Y$;
(e) $\left(\nabla_{X} \stackrel{*}{A_{\xi}}\right)(Y)-\left(\nabla_{Y} \stackrel{*}{A_{\xi}}\right)(X)=\tau(Y) \stackrel{*}{A_{\xi}} X-\tau(X) \stackrel{*}{A_{\xi}} Y-2 d \tau(X, Y) \xi$;
(f) $\nabla_{X} P Z=\nabla_{X} Z-X \cdot \eta(Z) \xi+\eta(Z) \stackrel{*}{A_{\xi}}+\eta(Z) \tau(X) \xi$.

Now, we recall the definition of a screen conformal lightlike hypersurface of a semi-Riemannian manifold $\bar{M}$.

Definition 3.1. ([1]). A lightlike hypersurface $(M, g, S(T M))$ of a semi-Riemannian manifold $\bar{M}$ is said to be screen globally) conformal if the shape operators $A_{N}$ and $\stackrel{*}{A}$ of $M$ and its screen distribution $S(T M)$ are related by

$$
A_{N}=\varphi \stackrel{*}{A} \xi
$$

where $\varphi$ is a non-vanishing smooth function on a neighborhood $\mathcal{U}$ in $M$. In case $\mathcal{U}=M$, the screen conformality is said to be global.

It is easy to see that (3.1) is equivalent to

$$
C(Y, P Z)=\varphi B(Y, Z),
$$

for all $X, Y \in \Gamma(T M)$.

We note that there are many examples of screen conformal lightlike hypersurfaces of semi-Riemannian manifolds see [1]

Next, a screen shape operator $\stackrel{*}{A} \xi$ is said to be parallel if $\nabla \stackrel{*}{A} \xi=0$ i.e.

$$
\nabla X \stackrel{*}{A} \xi=\stackrel{*}{A_{\xi}}\left(\nabla_{X} Y\right),(\nabla \text { and } \stackrel{*}{A} \xi \text { commute })
$$

for all $X, Y \in \Gamma(T M)$.

In the sequel, we consider a lightlike hypersurface $M$ of $(m+2)$-dimensional Lorentz manifold $(\bar{M}(k), \bar{g})$ of constant curvature $k$ and we suppose that the screen shape operator $\stackrel{*}{A}$ is parallel. Then we get

$$
\begin{aligned}
R(X, Y)\left(\stackrel{*}{A_{\xi}} Z\right) & =\nabla_{X} \nabla_{Y}\left(\stackrel{*}{A_{\xi}} Z\right)-\nabla_{Y} \nabla_{X}\left(\stackrel{*}{A}_{\xi} Z\right)-\nabla_{[X, Y]}\left(\stackrel{*}{A_{\xi}} Z\right) \\
& =\nabla_{X} \stackrel{*}{A}_{\xi}\left(\nabla_{Y} Z\right)-\nabla_{Y} \stackrel{*}{A}_{\xi}\left(\nabla_{X} Z\right)-\stackrel{*}{A}_{\xi}\left(\nabla_{[X, Y]} Z\right) \\
& =\stackrel{*}{A}_{\xi}\left(\nabla_{X} \nabla_{Y} Z\right)-\stackrel{*}{A}\left(\nabla_{Y} \nabla_{X} Z\right)-\stackrel{*}{A}\left(\nabla_{[X, Y]} Z\right) \\
& =\stackrel{*}{A}_{\xi} R(X, Y) Z,
\end{aligned}
$$

Thus, we have

$$
R(X, Y)\left(\stackrel{*}{A_{\xi}} Z\right)=\stackrel{*}{A} \xi R(X, Y) Z .
$$


Using (1) in Proposition 3.1, we have

$$
\begin{array}{rll}
R(X, Y)\left(\stackrel{*}{A_{\xi}} Z\right)= & k\left\{g\left(Y, \stackrel{*}{A_{\xi}} Z\right) X-g\left(X, \stackrel{*}{A_{\xi}} Z\right) Y\right\} \\
- & B\left(X, \stackrel{*}{A}_{\xi} Z\right) A_{N} Y+B\left(Y, \stackrel{*}{A_{\xi}} Z\right) A_{N} X \\
\stackrel{(2.19)}{=} & k\left\{g(Y, \stackrel{*}{A} Z) X-g\left(X, \stackrel{*}{A_{\xi}} Z\right) Y\right\} \\
- & g\left(\stackrel{*}{A}_{\xi} X, \stackrel{*}{A}_{\xi} Z\right) A_{N} Y+g\left(\stackrel{*}{A_{\xi}} Y, \stackrel{*}{A} \xi\right) A_{N} X .
\end{array}
$$

On the other hand, we have by using (1) in Proposition 3.1

$$
\begin{aligned}
\stackrel{*}{A} \xi(R(X, Y) Z) & =k\left\{g(Y, Z) \stackrel{*}{A_{\xi}} X-g(X, Z) \stackrel{*}{A_{\xi}} Y\right\} \\
& -g\left(\stackrel{*}{A}_{\xi} X, Z\right) \stackrel{*}{A_{\xi}} A_{N} Y+g\left(\stackrel{*}{A_{\xi}} Y, Z\right) \stackrel{*}{A_{\xi}} A_{N} X .
\end{aligned}
$$

Then, using (3.4), (3.5) and (3.6), we obtain

$$
\begin{aligned}
& k\left\{g\left(Y, \stackrel{*}{A}_{\xi} Z\right) X-g\left(X, \stackrel{*}{A_{\xi}} Z\right) Y\right\}-g\left(\stackrel{*}{A}_{\xi} X, \stackrel{*}{A_{\xi}} Z\right) A_{N} Y \\
& +g\left(\stackrel{*}{A_{\xi}} Y, \stackrel{*}{A_{\xi}} Z\right) A_{N} X \\
= & k\left\{g(Y, Z) \stackrel{*}{A_{\xi}} X-g(X, Z) \stackrel{*}{A} \xi Y\right\}-g\left(\stackrel{*}{A_{\xi}} X, Z\right) \stackrel{*}{A_{\xi}} A_{N} Y \\
& +g\left(\stackrel{*}{A_{\xi}} Y, Z\right) \stackrel{*}{A_{\xi}} A_{N} X .
\end{aligned}
$$

Note that for a class of screen conformal lightlike hypersurface $M$ of a Lorentzian manifold, the screen distribution $S(T M)$ is Riemannian, integrable and the induced Ricci tensor on $M$ is symmetric ([1]). Then, according to Proposition 3.4 in [9], there exists a canonical null pair $\{\xi, N\}$ satisfying (2.2) such that the corresponding 1 -form $\tau$ from $(2.9)$ vanishes. Since $\xi$ is an eigenvector field of $\stackrel{*}{A}$ corresponding to the eigenvalue 0 and $\stackrel{*}{A}$ 位 $\Gamma(S(T M))$-valued real symmetric, $\stackrel{*}{A}$ has $m$ orthonormal eigenvector fields in $S(T M)$ and is diagonalizable. Consider a frame field of eigenvectors $\left\{\xi, E_{1}, \ldots, E_{m}\right\}$ of $\stackrel{*}{A}_{\xi}$ such that $\left\{E_{1}, \ldots, E_{m}\right\}$ is an orthonormal frame field of $S(T M)$. Then, $\stackrel{*}{A} E_{i}=\lambda_{i} E_{i}, \quad 1 \leq i \leq m$. We call the eigenvalues $\lambda_{i}$ the screen principal curvatures for all $i$.

Using (3.4), we have for $1 \leq i, j \leq m$ and $i \neq j$

$$
R\left(E_{i}, E_{j}\right)\left(\stackrel{*}{A} E_{\xi}\right)=\stackrel{*}{A}{ }_{\xi} R\left(E_{i}, E_{j}\right) E_{j} .
$$

Thus, from (3.7) we have

$$
k \lambda_{j} E_{i}+\lambda_{j}^{2} A_{N} E_{i}=k \lambda_{i} E_{i}+\lambda_{j} \stackrel{*}{A_{\xi}} A_{N} E_{i},
$$

and then,

$$
\begin{aligned}
g\left(k \lambda_{j} E_{i}+\lambda_{j}^{2} A_{N} E_{i}, E_{i}\right) & =g\left(k \lambda_{i} E_{i}+\lambda_{j} \stackrel{*}{A_{\xi}} A_{N} E_{i}, E_{i}\right) \\
k \lambda_{j} g\left(E_{i}, E_{i}\right)+\lambda_{j}^{2} g\left(A_{N} E_{i}, E_{i}\right) & =k \lambda_{i} g\left(E_{i}, E_{i}\right)+\lambda_{j} g\left(\stackrel{*}{A_{\xi}} A_{N} E_{i}, E_{i}\right) \\
k \lambda_{j}+\lambda_{j}^{2} g\left(A_{N} E_{i}, E_{i}\right) & =k \lambda_{i}+\lambda_{j} g\left(A_{N} E_{i}, \stackrel{*}{A} E_{i}\right) \\
k \lambda_{j}+\lambda_{j}^{2} g\left(A_{N} E_{i}, E_{i}\right) & =k \lambda_{i}+\lambda_{j} \lambda_{i} g\left(A_{N} E_{i}, E_{i}\right) .
\end{aligned}
$$

We conclude,

$$
\left(\lambda_{j}-\lambda_{i}\right)\left(k+\lambda_{j} g\left(A_{N} E_{i}, E_{i}\right)\right)=0 .
$$


If the screen is conformal, we have $A_{N} E_{i}=\varphi \stackrel{*}{A} E_{\xi}=\varphi \lambda_{i} E_{i}$, then (3.8) becomes

$$
\left(\lambda_{j}-\lambda_{i}\right)\left(k+\varphi \lambda_{j} \lambda_{i}\right)=0
$$

Under the conditions of the equation (3.9), we now prove several Lemmas.

Lemma 3.1. Either $\operatorname{rank}_{A_{\xi}}^{*}=0$ or $\operatorname{rank} \stackrel{*}{A}_{\xi}=m$.

Proof. Since $\stackrel{*}{A} \xi \xi=0$, then $\operatorname{rank}_{\xi} \stackrel{*}{A}_{\xi}<m+1$. Assume that $\operatorname{rank}^{*} A_{\xi} \neq m$. Then for some $i$ we have $\lambda_{i}=0$ and using (3.9) it follows that $\lambda_{j}=0$. Thus all eigenvalues of $\stackrel{*}{A}$ are zero and $\operatorname{rank} \stackrel{*}{A} \xi=0$.

Lemma 3.2. If $\operatorname{rank} \stackrel{*}{A} \neq 0$, then $\stackrel{*}{A} \xi$ has at most two distinct screen principal curvatures.

Proof. For $i=i_{0}$, equation (3.9) becomes $\left(\lambda_{j}-\lambda_{i_{0}}\right)\left(k+\varphi \lambda_{i_{0}} \lambda_{j}\right)=0$. If $\lambda_{j} \neq \lambda_{i_{0}}$, then $\lambda_{j}=-\frac{k}{\varphi \lambda_{i_{0}}}$. Then $\stackrel{*}{A}$ has at most two distinct screen principal curvatures.

By the Lemma 3.2 it follows that $\stackrel{*}{A}$ g has at most two distinct screen principal curvatures, $\lambda$ and $\mu=-\frac{k}{\varphi \lambda}$.

Define two distributions:

$$
\begin{aligned}
& T_{\lambda}=\left\{X \in \Gamma(T M) \mid \stackrel{*}{A_{\xi}} X=\lambda P X\right\}, \\
& T_{\mu}=\left\{X \in \Gamma(T M) \mid \stackrel{*}{A} A_{\xi} X=\mu P X\right\} .
\end{aligned}
$$

Lemma 3.3. The distributions $T_{\lambda}$ and $T_{\mu}$ are both involutive. 
Proof. Let choose $X, Y \in \Gamma\left(T_{\lambda}\right)$, then

$$
\begin{aligned}
\stackrel{*}{A_{\xi}}[X, Y]= & \mathcal{*}_{\xi} \nabla_{X} Y-\stackrel{*}{A} \xi_{\xi} \nabla_{Y} \\
= & \nabla_{X} \stackrel{*}{A}_{\xi} Y-\left(\nabla_{X} \stackrel{*}{A}_{\xi}\right) Y-\nabla_{Y} \stackrel{*}{A_{\xi}} X+\left(\nabla_{Y} \stackrel{*}{A}_{\xi}\right) X \\
= & \nabla_{X} \stackrel{*}{A}_{\xi} Y-\nabla_{Y} \stackrel{*}{A}_{\xi} X \\
= & \nabla_{X}(\lambda P Y)-\nabla_{Y}(\lambda P X) \\
= & (X \cdot \lambda) P Y+\lambda \nabla_{X} P Y-(Y \cdot \lambda) P X-\lambda \nabla_{Y} P X \\
= & (X \cdot \lambda) P Y+\lambda \nabla_{X}[Y-\eta(Y) \xi]-(Y \cdot \lambda) P X-\lambda \nabla_{Y}[X-\eta(X) \xi] \\
= & (X \cdot \lambda) P Y+\lambda\left[\nabla_{X} Y-X \cdot \eta(Y) \xi-\eta(Y) \nabla_{X} \xi\right]-(Y \cdot \lambda) P X \\
& -\lambda\left[\nabla_{Y} X-Y \cdot \eta(X) \xi-\eta(X) \nabla_{Y} \xi\right] \\
= & (X \cdot \lambda) P Y+\lambda\left[\nabla_{X} Y-X \cdot \eta(Y) \xi-\eta(Y)\left(-\stackrel{*}{A}_{\xi} X-\tau(X) \xi\right)\right] \\
& -(Y \cdot \lambda) P X-\lambda\left[\nabla_{Y} X-Y \cdot \eta(X) \xi-\eta(X)\left(-\stackrel{*}{A}_{\xi} Y-\tau(Y) \xi\right)\right] \\
= & (X \cdot \lambda) P Y+\lambda\left[\nabla_{X} Y-X \cdot \eta(Y) \xi-\eta(Y)(-\lambda P X-\tau(X) \xi)\right] \\
& -(Y \cdot \lambda) P X-\lambda\left[\nabla_{Y} X-Y \cdot \eta(X) \xi-\eta(X)(-\lambda P Y-\tau(Y) \xi)\right] \\
= & (X \cdot \lambda) P Y-(Y \cdot \lambda) P X+\lambda\left(\nabla X Y-\nabla_{Y} X\right)+\lambda^{2}(\eta(Y) P X \\
- & \eta(X) P Y)+\lambda[-X \cdot \eta(Y)+Y \cdot \eta(X)+\tau(X) \eta(Y)-\tau(Y) \eta(X)] \xi \\
= & (X \cdot \lambda) P Y-(Y \cdot \lambda) P X+\lambda[X, Y]+\lambda^{2}(\eta(Y) P X-\eta(X) P Y) \\
& \lambda[-X \cdot \eta(Y)+Y \cdot \eta(X)+\tau(X) \eta(Y)-\tau(Y) \eta(X)] \xi \\
= & (X \cdot \lambda) P Y-(Y \cdot \lambda) P X+\lambda P[X, Y]+\lambda \eta([X, Y]) \xi \\
& +\lambda^{2}(\eta(Y) P X-\eta(X) P Y)+\lambda[-X \cdot \eta(Y)+Y \cdot \eta(X) \\
& +\tau(X) \eta(Y)-\tau(Y) \eta(X)] \xi
\end{aligned}
$$

Now, we compute:

$$
\begin{aligned}
\eta([X, Y])= & \eta\left(\nabla_{X} Y-\nabla_{Y} X\right)=\bar{g}\left(\nabla_{X} Y-\nabla_{Y} X, N\right) \\
= & \bar{g}\left(\nabla_{X} Y, N\right)-\bar{g}\left(\nabla_{Y} X, N\right) \\
= & X \cdot \bar{g}(Y, N)-\bar{g}\left(Y, \nabla_{X} N\right)-Y \cdot \bar{g}(X, N)+\bar{g}\left(X, \nabla_{Y} N\right) \\
= & X \cdot \bar{g}(Y, N)-\bar{g}\left(Y,-A_{N}(X)+\tau(X) N\right)-Y \cdot \bar{g}(X, N) \\
& +\bar{g}\left(X,-A_{N}+\tau(Y) N\right) \\
= & X \cdot \bar{g}(Y, N)-\bar{g}\left(Y,-A_{N}(X)\right)-\tau(X) \bar{g}(Y, N) \\
- & Y \cdot \bar{g}(X, N)+\bar{g}\left(X,-A_{N}(Y)\right)+\tau(Y) \bar{g}(X, N) \\
= & X \cdot \bar{g}(Y, N)-\bar{g}\left(Y,-A_{N}(X)\right)-Y \cdot \bar{g}(X, N) \\
+ & \bar{g}\left(X,-A_{N}(Y)\right)-\tau(X) \eta(Y)+\tau(Y) \eta(X) \\
= & X \cdot \eta(Y)-\varphi \bar{g}\left(Y,-\stackrel{*}{A_{\xi}}(X)\right)-Y \cdot \eta(X) \\
+ & \varphi \bar{g}\left(X,-\stackrel{A}{A}_{\xi}(Y)\right)-\tau(X) \eta(Y)+\tau(Y) \eta(X) .
\end{aligned}
$$


Then,

$$
\begin{aligned}
\eta([X, Y])= & X \cdot \eta(Y)+\varphi \lambda \bar{g}(Y, P X)-Y \cdot \eta(X)-\varphi \lambda \bar{g}(X, P Y) \\
& -\tau(X) \eta(Y)+\tau(Y) \eta(X) \\
= & X \cdot \eta(Y)+\varphi \lambda \bar{g}(Y, X)-Y \cdot \eta(X)-\varphi \lambda \bar{g}(X, Y) \\
& -\tau(X) \eta(Y)+\tau(Y) \eta(X) \\
= & X \cdot \eta(Y)-Y \cdot \eta(N)-\tau(X) \eta(Y)+\tau(Y) \eta(X) .
\end{aligned}
$$

Using (3.10) and (3.11) we get,

$$
\stackrel{*}{A}_{\xi}[X, Y]=(X \cdot \lambda) P Y-(Y \cdot \lambda) P X+\lambda P[X, Y]+\lambda^{2}(\eta(Y) P X-\eta(X) P Y) .
$$

Then

$$
\left(\stackrel{*}{A}_{\xi}-\lambda P\right)[X, Y]=(X \cdot \lambda) P Y-(Y \cdot \lambda) P X+\lambda^{2}(\eta(Y) P X-\eta(X) P Y) .
$$

However, the left-hand member of (3.12) belongs to $T_{\lambda}$. In fact,

$$
[X, Y]=[X, Y]_{\lambda}+[X, Y]_{\mu}+\eta([X, Y]) \xi
$$

implies that

$$
\begin{aligned}
(\stackrel{*}{A} \xi-\lambda P)[X, Y]= & \left(\stackrel{*}{A}_{\xi}-\lambda P\right)\left([X, Y]_{\lambda}+[X, Y]_{\mu}+\eta([X, Y]) \xi\right) \\
= & \stackrel{*}{A_{\xi}}[X, Y]_{\lambda}-\lambda P[X, Y]_{\lambda}+\stackrel{*}{A} \xi[X, Y]_{\mu} \\
& -\lambda P[X, Y]_{\mu}+\eta([X, Y]) \stackrel{*}{A} \xi \\
= & (\mu-\lambda)[X, Y]_{\mu} .
\end{aligned}
$$

On the other hand, the right-hand member of (3.12) belongs to $T_{\lambda}$ and therefore

$$
\begin{aligned}
& \left(\stackrel{*}{A_{\xi}}-\lambda P\right)[X, Y]=0, \\
& (X \cdot \lambda) P Y-(Y \cdot \lambda) P X+\lambda^{2}(\eta(Y) P X-\eta(X) P Y)=0 .
\end{aligned}
$$

Hence $(\stackrel{*}{A} \xi-\lambda P)[X, Y]=0$, thus $[X, Y] \in \Gamma\left(T_{\lambda}\right)$. This shows that the distribution $T_{\lambda}$ is involutive. Using the same way, we can see that the distribution $T_{\mu}$ is also involutive.

Define two distributions $T_{\lambda}^{s}=T_{\lambda} \cap S(T M)$ and $T_{\mu}^{s}=T_{\mu} \cap S(T M)$. Since $\eta(X)=0$ for all $X, Y \in \Gamma\left(T_{\lambda}^{s}\right)$, equations (3.13) become

$$
(\stackrel{*}{A}-\lambda)[X, Y]=0, \quad(X \cdot \lambda) Y-(Y \cdot \lambda) X=0
$$

We have the following Lemma.

Lemma 3.4. If $\operatorname{dim} T_{\lambda}^{s}>1$, then $X \cdot \lambda=0, \tau(X)=0, X \cdot \varphi=0$ and $X \cdot \mu=0$, for all $X \in \Gamma\left(T_{\lambda}^{s}\right)$.

Proof. Let $X, Y \in \Gamma\left(T_{\lambda}^{s}\right)$. If $\operatorname{dim} T_{\lambda}^{s}>1$, we can choose $X, Y$ to be linearly independent. Thus, using the right equation in (3.14) we have $X \cdot \lambda$.

Since ${ }^{*} \xi$ is parallel, by using (5) in Proposition 3.1, we have the following: $\lambda \tau(Y) X-$ $\lambda \tau(X) Y-2 d \tau(X, Y) \xi=0$. Then $\lambda \tau(Y) X-\lambda \tau(X) Y=0$ and $d \tau(X, Y)=0$. Since $\lambda \neq 0$, we can again choose $X, Y$ to be linearly independent, then $\tau(X)=0$. Using (4) in Proposition 3.1, (3.1) and $\tau=0$, we have $(Y \cdot \varphi) X-(X \cdot \varphi) Y=0$, by linearly independent we have $X \cdot \varphi=0$. 
Since $\mu=-\frac{k}{\varphi \lambda}$, it follows that $X \cdot \mu=-k \frac{(X \cdot \varphi) \lambda+(X \cdot \lambda) \varphi}{(\varphi \lambda)^{2}}$, and this completes the proof.

Lemma 3.5. For $X \in \Gamma\left(T_{\lambda}^{s}\right), Y \in \Gamma\left(T_{\mu}^{s}\right)$, we have $\nabla_{X} Y \in \Gamma\left(T_{\mu}^{s}\right)$ and $\nabla_{Y} X \in$ $\Gamma\left(T_{\lambda}^{s}\right)$.

Proof. Let $X \in \Gamma\left(T_{\lambda}^{S}\right), Y \in \Gamma\left(T_{\mu}^{s}\right)$. We have

$$
\begin{aligned}
\left(\nabla_{X} \stackrel{*}{A_{\xi}}\right) Y & =\nabla_{X}\left(\stackrel{*}{A}_{\xi} Y\right)-\stackrel{*}{A}_{\xi}\left(\nabla_{X} Y\right) \\
& =\nabla_{X}(\mu Y)-\stackrel{*}{A_{\xi}}\left(\nabla_{X} Y\right) \\
& =(X \cdot \mu) Y+\mu \nabla_{X} Y-\stackrel{*}{A_{\xi}} \nabla_{X} Y \\
& =\mu \nabla_{X} Y-\stackrel{*}{A_{\xi}}\left(\nabla_{X} Y\right)
\end{aligned}
$$

Since $\nabla_{X} \stackrel{*}{A_{\xi}}=0$, we have $\stackrel{*}{A_{\xi}}\left(\nabla_{X} Y\right)=\mu \nabla_{X} Y$ which proves that $\nabla_{X} Y$ belongs to $\Gamma\left(T_{\mu}^{s}\right)$. Using the same argument, we see that $\nabla_{Y} X$ belongs to $\Gamma\left(T_{\lambda}^{s}\right)$.

Lemma 3.6. $T_{\lambda}^{s}$ and $T_{\mu}^{s}$ are totally geodesic and parallel distributions.

Proof. By Lemma 3.5, if $X \in \Gamma\left(T_{\lambda}^{s}\right), Y \in \Gamma\left(T_{\mu}^{s}\right)$, we have $\nabla_{X} Y \in \Gamma\left(T_{\mu}^{s}\right)$ and $\nabla_{Y} X \in \Gamma\left(T_{\lambda}^{s}\right)$ which shows that $T_{\lambda}^{s}$ and $T_{\mu}$ are parallel. Let $X, Z \in \Gamma\left(T_{\lambda}^{s}\right), Y \in$ $\Gamma\left(T_{\mu}^{s}\right)$. Since $\eta(Y)=\eta(Z)=0$,

$$
g\left(\nabla_{Z} X, Y\right)+g\left(X, \nabla_{Z} Y\right)=Z \cdot g(X, Y)=0 .
$$

By Lemma 3.5, $\nabla_{Z} Y \in \Gamma\left(T_{\mu}^{s}\right)$ implies that $g\left(Y, \nabla_{Z} X\right)=0$. Then $g\left(Y, \nabla_{Z} X\right)=$ $g\left(Y, \stackrel{*}{\nabla}_{Z} X+C(X, Z) \xi\right)=g\left(Y, \stackrel{*}{\nabla}_{Z} X\right)=0$. It follows $\stackrel{*}{\nabla}_{Z} X \in \Gamma\left(T_{\lambda}^{s}\right)$ for all $Z$ and $X$ in $\Gamma\left(T_{\lambda}^{s}\right)$ which shows that $T_{\lambda}^{s}$ is totally geodesic. By the same way, we can see that $T_{\mu}$ is totally geodesic.

Next, we say that $M$ is totally umbilical if there exists a smooth function $\rho$ such that

$$
B(X, Y)=\rho g(X, Y)
$$

for all $X, Y \in \Gamma(T M)$, or equivalently,

$$
\stackrel{*}{A}{ }_{\xi}=\rho P X,
$$

for all $X \in \Gamma(T M)$.

$M$ is said to be a totally geodesic lightlike hypersurface if the second fundamental form $B=0$ or equivalently $\stackrel{*}{A}=0$. Now we prove the following theorem.

Theorem 3.1. Let $(M, g, S(T M))$ be a lightlike hypersurface of $(m+2)$-dimensional Lorentz manifold $(\bar{M}(k), \bar{g})$ of constant curvature $k$ such that the screen shape operator is parallel. Then,

(a) $M$ is either totally geodesic or totally umbilic;

(b) if the screen is conformal, $M$ is a locally lightlike triple product manifold $C \times\left(M^{\prime}=M_{\lambda} \times M_{\mu}\right)$, where $C$ is a null curve, $M^{\prime}$ is an integral manifold of $S(T M), M_{\lambda}$ and $M_{\mu}$ are leaves of some distributions of $M$ such that they are totally geodesic (in $S(T M)$ ) Riemannian manifolds of constant curvature $\left(k+2 \varphi \lambda^{2}\right)$ and $\left(k+2 \varphi \mu^{2}\right)$ respectively. 
Proof. (a) From (3.8), we have $\left(\lambda_{j}-\lambda_{i}\right)=0$ or $\left(k+\lambda_{j} g\left(A_{N} E_{i}, E_{i}\right)\right)=0$.

If $\left(\lambda_{j}-\lambda_{i}\right)=0$, then, $\lambda_{i}=\lambda_{j}$ for any $i, j$. This means that all eigenfuntions are equal. We note it by $\lambda$. Let $X \in \Gamma(T M)$, we have

$$
\begin{aligned}
\stackrel{*}{A} X^{\prime} & =\stackrel{*}{A}\left(\sum_{i=1}^{m} X_{i} E_{i}+\eta(X) \xi\right)=\sum_{i=1}^{m} X_{i} \stackrel{*}{A_{\xi}} E_{i}+\eta(X) \stackrel{*}{A_{\xi}} \xi \\
& =\sum_{i=1}^{m} X_{i} \lambda_{i} E_{i}=\lambda \sum_{i=1}^{m} X_{i} E_{i} .
\end{aligned}
$$

Then $\stackrel{*}{A} X=\lambda P X$. If $\lambda=0, M$ is totally geodesic and if not $M$ is totally umbilic.

(b)From ([1]) a conformal lightlike hypersurface $M$ is locally a product manifold $C \times M^{\prime}$, where $C$ is a null curve and $M^{\prime}$ is a leaf of $S(T M)$. Since the leaf $M^{\prime}$ of $S(T M)$ is Riemannian and $S(T M)=T_{\lambda}^{s} \oplus_{\text {orth }} T_{\mu}^{s}$, where $T_{\lambda}^{s}$ and $T_{\mu}^{s}$ are parallel distributions with respect to the induced connection ${ }^{*}$ of $M^{\prime}$, by the decomposition theorem of de Rham ([8]) we have $M^{\prime}=M_{\lambda} \times M_{\mu}$, where $M_{\lambda}$ and $M_{\mu}$ are some leaves of $T_{\lambda}^{s}$ and $T_{\mu}^{s}$, respectively. It follows that $M=C \times M^{\prime}=C \times M_{\lambda} \times M_{\mu}$. Let $X, Y, Z, W \in \Gamma\left(T_{\lambda}^{s}\right)$. Using (1) in Proposition (3.1) and equations (2.19) and (3.1) we have

$$
\begin{aligned}
g(R(X, Y) Z, W)= & k\{g(Y, Z) g(X, W)-g(X, Z) g(Y, W)\} \\
- & \varphi g\left(\stackrel{*}{\xi}_{\xi} X, Z\right) g\left({ }^{*} A_{\xi} Y, W\right)+\varphi g\left({ }^{*} Y, Z\right) g\left({ }^{*} X, W\right) \\
= & k\{g(Y, Z) g(X, W)-g(X, Z) g(Y, W)\} \\
& -\varphi \lambda^{2} g(X, Z) g(Y, W)+\varphi \lambda^{2} g(Y, Z) g(X, W) \\
= & \left(k+\varphi \lambda^{2}\right) g(g(Y, Z) X-g(X, Z) Y, W) .
\end{aligned}
$$

Again, by using (3.1), (3.2), (2.19), (2.20), (2.22) and (3.17), we have

$$
\begin{aligned}
& \left(k+\varphi \lambda^{2}\right) g(g(Y, Z) X-g(X, Z) Y, W)= \\
& g(\stackrel{*}{R}(X, Y) Z, W)-\varphi \lambda^{2} g(g(Y, Z) X-g(X, Z) Y, W) .
\end{aligned}
$$

Then, $\stackrel{*}{R}(X, Y) Z=\left(k+2 \varphi \lambda^{2}\right)\{g(Y, Z) X-g(X, Z) Y\}$, for all $X, Y, Z$ in $\Gamma\left(T_{\lambda}^{s}\right)$. Thus $M_{\lambda}$ is a Riemannian manifold of constant curvature $\left(k+2 \varphi \lambda^{2}\right)$. In the same way we obtain that $M_{\mu}$ is a Riemannian manifold of constant curvature $\left(k+2 \varphi \mu^{2}\right)$.

Theorem 3.2. Let $M_{\lambda}$ and $M_{\mu}$ be as in the theorem 3.1 and $\operatorname{dim}\left(M_{\lambda}\right)=r$. Then $M_{\lambda}$ and $M_{\mu}$ are totally umbilical submanifolds of $\bar{M}(k)$ of codimension $(m-r+2)$ and $(r+2)$, respectively.

Proof. Let $i$ be the immersion of $M_{\lambda}$ in $M$ and $M^{\prime}$ be a leaf of $S(T M)$. Consider in the normal bundle $T M^{\perp}$, the vector fields

$$
\zeta_{1}=\frac{\varphi}{\sqrt{2|\varphi|}} \xi+\frac{1}{\sqrt{2|\varphi|}} N \text { and } \zeta_{2}=\frac{\varphi}{\sqrt{2|\varphi|}} \xi-\frac{1}{\sqrt{2|\varphi|}} N .
$$


Clearly, $\left\{\zeta_{1}, \zeta_{2}\right\}$ is an orthonormal basis, where $\zeta_{1}$ and $\zeta_{2}$ are spacelike and timelike respectively. Then, for any $X, Y \in \Gamma\left(T M_{\lambda}\right)$, we have

$$
\begin{aligned}
\bar{\nabla}_{X} Y & =\nabla_{X}^{\lambda} Y+h^{\lambda}(X, Y) \\
& =\nabla_{X}^{\lambda} Y+\sum_{a=r+1}^{m+2} g_{\lambda}\left(A_{\xi_{a}^{\lambda}} X, Y\right) \xi_{a}^{\lambda},
\end{aligned}
$$

where $g_{\lambda}, \nabla^{\lambda}$ are the induced metric and induced connection of $M_{\lambda}$ respectively, $\xi_{a}^{\lambda}$ are orthonormal normal to $M_{\lambda}$ in $\bar{M}(k)$ such that $\xi_{m+1}^{\lambda}=\zeta_{1}$ and $\xi_{m+2}^{\lambda}=\zeta_{2}, A_{\xi_{a}^{\lambda}}$ are corresponding shape operators of $\xi_{a}^{\lambda}$ and $h^{\lambda}$ is the second fundamental form of $M_{\lambda}$ in $\bar{M}(k)$. On the other hand, we have

$$
\begin{aligned}
\bar{\nabla}_{X} Y & =\nabla_{X} Y+B(X, Y) N=\nabla_{X} Y+g\left({ }^{*} A_{\xi} X, Y\right) N \\
& ={ }^{*} \nabla_{X} Y+C(X, Y) \xi+g\left(\stackrel{*}{A}_{\xi} X, Y\right) N \\
& ={ }^{*} \nabla_{X} Y+g\left(A_{N} X, Y\right) \xi+g\left({ }^{*} A_{\xi} X, Y\right) N \\
& ={ }^{*} X Y+\varphi g\left(\stackrel{*}{A}_{\xi} X, Y\right) \xi+g\left({ }^{A} A_{\xi} X, Y\right) N \\
& =\nabla_{X}^{\lambda} Y+h_{M^{\prime}}^{\lambda}(X, Y)+g\left({ }^{*} A_{\xi} X, Y\right)(\varphi \xi+N) \\
& =\nabla_{X}^{\lambda} Y+h_{M^{\prime}}^{\lambda}(X, Y)+\lambda g(X, Y)(\varphi \xi+N),
\end{aligned}
$$

where $h_{M^{\prime}}^{\lambda}$ is the second fundamental form of $M_{\lambda}$ is $S(T M)$. By Lemma 3.6, $M_{\lambda}$ is totally geodesic in $S(T M)$, and consequently the last equation can be written as

$$
\bar{\nabla}_{X} Y=\nabla_{X}^{\lambda} Y+\lambda g(X, Y)(\varphi \xi+N)=\nabla_{X}^{\lambda} Y+\sqrt{2|\varphi|} \lambda g(X, Y) \zeta_{1} .
$$

Comparing (3.18) and (3.19), we have $A_{\xi_{a}^{\lambda}} X=0$ for all $a \neq m+1$ and $A_{\xi_{m+1}^{\lambda}} X=$ $A_{\zeta_{1}} X=\sqrt{2|\varphi|} \lambda X$. Thus, $M_{\lambda}$ is a totally umbilical submanifold of $\bar{M}(k)$. Similarly, we can prove that $M_{\mu}$ is a totally umbilical submanifold in $\bar{M}(k)$.

Let us change $\tilde{\xi}=\alpha \xi$, then $\stackrel{*}{A_{\tilde{\xi}}}=\alpha \stackrel{*}{A_{\xi}}$, where $\alpha$ is a non-zero smooth function. By direct calculation we have,

$$
\left(\nabla_{X} \stackrel{*}{A_{\tilde{\xi}}}\right) Y=(X \cdot \alpha) \stackrel{*}{A_{\xi}} Y+\alpha\left(\nabla_{X} \stackrel{*}{A_{\xi}}\right) Y .
$$

We prove the following

Proposition 3.2. Let $M$ be a lightlike hypersurface of a semi-Riemannian manifold $(\bar{M}, \bar{g})$. Let $\xi \in \Gamma(\operatorname{Rad}(T M))$ and make a change $\tilde{\xi}=\alpha \xi$. If $\stackrel{*}{A_{\xi}}$ is parallel, then

$$
R(X, Y) \stackrel{*}{A_{\tilde{\xi}}} Z=\stackrel{*}{A}_{\widetilde{\xi}} R(X, Y) Z .
$$

for any $X, Y Z \in \Gamma(T M)$. Moreover, $\stackrel{*}{\widetilde{\xi}}_{\tilde{\xi}}$ is parallel if and only if $\alpha$ is constant. 
Proof. From the definition of curvature tensor, it follows

$$
\begin{aligned}
& R(X, Y) \stackrel{*}{A}_{\widetilde{\xi}} Z=\nabla_{X} \nabla_{Y} \stackrel{*}{A}_{\widetilde{\xi}} Z-\nabla_{Y} \nabla_{X} \stackrel{*}{A}_{\widetilde{\xi}} Z-\nabla_{[X, Y]} \stackrel{*}{A}_{\widetilde{\xi}} Z \\
& =\nabla_{X}\left(\stackrel{*}{A}_{\widetilde{\xi}}\left(\nabla_{Y} Z\right)+\left(\nabla_{Y} \stackrel{*}{A_{\widetilde{\xi}}}\right) Z\right)-\nabla_{Y}\left(\stackrel{*}{A_{\widetilde{\xi}}}\left(\nabla_{X} Z\right)\right. \\
& \left.+\left(\nabla_{X} \stackrel{*}{A}_{\tilde{\xi}}\right) Z\right)-\stackrel{*}{A}_{\tilde{\xi}}\left(\nabla_{[X, Y]} Z\right)-\left(\nabla_{[X, Y]} \stackrel{*}{A}_{\tilde{\xi}}\right) Z \\
& \stackrel{(3.20)}{=} \nabla_{X}\left(\stackrel{*}{A_{\tilde{\xi}}}\left(\nabla_{Y} Z\right)\right)+(X Y \cdot \alpha) \stackrel{*}{A_{\xi}} Z+(Y \cdot \alpha) \nabla_{X} \stackrel{*}{A_{\xi}} Z \\
& +\nabla_{X}\left(\alpha\left(\nabla_{Y} A_{\xi}\right) Z\right)-\nabla_{X}\left(\stackrel{*}{A_{\tilde{\xi}}}\left(\nabla_{X} Z\right)\right)-(Y X \cdot \alpha) \stackrel{*}{A_{\xi}} Z \\
& -(X \cdot \alpha) \nabla_{Y} \stackrel{*}{A_{\xi}} Z-\nabla_{Y}\left(\alpha\left(\nabla_{X} A_{\xi}\right) Z\right)-\stackrel{*}{A}_{\tilde{\xi}}\left(\nabla_{[X, Y]} Z\right) \\
& -\quad([X, Y] \cdot \alpha) \stackrel{*}{A_{\xi}} Z-\left(\nabla_{[X, Y]} \stackrel{*}{A}\right) Z \\
& =\nabla_{X}\left(\stackrel{*}{A}_{\tilde{\xi}}\left(\nabla_{Y} Z\right)\right)-\nabla_{X}\left(\stackrel{*}{A}_{\widetilde{\xi}}\left(\nabla_{X} Z\right)\right)-\stackrel{*}{A}_{\widetilde{\xi}}\left(\nabla_{[X, Y]} Z\right) \\
& +(Y \cdot \alpha) \nabla_{X} \stackrel{*}{A} \xi Z-(X \cdot \alpha) \nabla_{Y} \stackrel{*}{A} \xi \\
& =\stackrel{*}{A}_{\widetilde{\xi}}\left(\nabla_{X} \nabla_{Y} Z\right)+\left(\nabla_{X} \stackrel{*}{A}_{\widetilde{\xi}}\right) \nabla_{Y} Z-\stackrel{*}{A}_{\widetilde{\xi}}\left(\nabla_{Y} \nabla_{X} Z\right) \\
& -\left(\nabla_{Y} \stackrel{*}{A}_{\widetilde{\xi}}\right) \nabla_{X} Z-\stackrel{*}{A}_{\widetilde{\xi}}\left(\nabla_{[X, Y]} Z\right) \\
& +(Y \cdot \alpha) \stackrel{*}{A} \xi \nabla_{X} Z-(X \cdot \alpha) \stackrel{*}{A_{\xi}} \nabla_{Y} Z \\
& \stackrel{(3.20)}{=} \stackrel{*}{A}_{\tilde{\xi}}\left(\nabla_{X} \nabla_{Y} Z\right)+(X \cdot \alpha) \stackrel{*}{A_{\xi}} \nabla_{Y} Z-\stackrel{*}{A_{\widetilde{\xi}}}\left(\nabla_{Y} \nabla_{X} Z\right) \\
& -(Y \cdot \alpha) \stackrel{*}{A_{\xi}} \nabla_{X} Z-\stackrel{*}{A}_{\widetilde{\xi}}\left(\nabla_{[X, Y]} Z\right) \\
& +(Y \cdot \alpha) \stackrel{*}{A} \nabla_{\xi} \nabla_{X} Z-(X \cdot \alpha) \stackrel{*}{A_{\xi}} \nabla_{Y} Z \\
& =\stackrel{*}{A}_{\widetilde{\xi}}\left(\nabla_{X} \nabla_{Y} Z-\nabla_{Y} \nabla_{X} Z-\nabla_{[X, Y]} Z\right) \\
& =\quad A_{\widetilde{\xi}}^{*} R(X, Y) Z \text {. }
\end{aligned}
$$

From equation (3.20), it is obvious that if $\stackrel{*}{A}_{\xi}$ is parallel, then $\stackrel{*}{A}_{\widetilde{\xi}}$ is parallel if and only if $X \cdot \alpha=0$ for all $X \in \Gamma(T M)$, that is if and only if $\alpha$ is constant.

Remark 3.1. Consider a frame field of eigenvectors $\left\{\xi, E_{1}, \ldots, E_{m}\right\}$ of ${ }_{A_{\tilde{\xi}}}^{*}$ such that $\left\{E_{1}, \ldots, E_{m}\right\}$ is an orthonormal frame field of $S(T M)$. If $\lambda_{i}$ is an eigenfuntion of $\stackrel{*}{A}$, then $\tilde{\lambda_{i}}=\alpha \lambda_{i}$ is an eigenfuntion of ${\stackrel{*}{A_{\tilde{\xi}}}}$. Thus, by using (1) in Proposition 3.1 and equation (3.21), we get

$$
\begin{aligned}
& \left(\tilde{\lambda_{j}}-\tilde{\lambda_{i}}\right)\left[k+\tilde{\lambda_{j}} g\left(\frac{1}{\alpha} A_{N} E_{i}, E_{i}\right)\right]= \\
& \alpha\left(\lambda_{j}-\lambda_{i}\right)\left[k+\lambda_{j} g\left(A_{N} E_{i}, E_{i}\right)\right]=0 .
\end{aligned}
$$

Then Eq.(3.8) does not depend on the choice of the null section $\xi$ of $\operatorname{Rad}(\mathrm{TM})$. Since results in theorem 3.1 and Theorem 3.2 are based on this equation, then 
Proposition 3.2 prove that these results are stable with any change of null section $\xi \in \operatorname{Rad}(T M)$.

\section{REFERENCES}

[1] Cyriaque Atindogbe and Duggal, Krishan L., Conformal screen on lightlike hypersurfaces. Int. J. of Pure and Applied Math., 11(2004), 421-442.

[2] Cyriaque A., Mahamane Mahi H. and Joël T., Lightlike Hypersurfaces in Lorentzian manifolds with constant screen principal curvatures. Afr. Diaspora J. Math, 16 (2014), no 2, pp $31-45$.

[3] Cyriaque A, Ezin J-P. and Joël T., Lightlike Einstein hypersurfaces in Lorentzian Manifolds with constant curvature. Kodai Math. Journal, vol. 29 No. 1, (2006) 58-71.

[4] Bejancu A., Ferradez A. and Lucas P., A new viewpoint on geometry of lightlike hypersurfaces in a semi-Euclidean space. Saitama Math. J. 16 (1998),31-38.

[5] Cartan E., Familles de surfaces isoparametriques dans les espaces a courbure constante, Ann. Mat. Pura IV 17, (1938) 177-191.

[6] Cartan E., Sur quelques familles remarquables d'hypersurfaces, C. R. Congrés Math. Liége (1939) 30-41; Oeuvres complètes Tome III, Vol. 2, 1481-1492.

[7] Chen B. Y., Geometry of submanifolds. Pure and Applied Mathematics, New York, DeKKer (1973).

[8] de Rham G., Sur la réductibilité d'un espace de Riemann, Comment. Math. Helv. 268, (1952) 328-344.

[9] Duggal, Krishan L. and Bejancu, A, Lightlike submanifolds of semi-Riemannian Manifolds and Applications. Kluwer Acad. Publishers, Dordrecht, volume 364, 1996.

[10] Duggal, Krishan L. and Jin D. H. A classification of Einstein lightlike hypersurfaces of a Lorentzian space form J. of Geomtry and Physics 60 (2010) 1881-1889.

Département de Mathématiques, Université Dan Dicko Dankoulodo de Maradi

E-mail address: hmahi2007@yahoo.fr/ mahi.harouna@imsp-uac.org 